\title{
Ecchymotic Purpura of the Breast Revealing a Locally Advanced Breast Cancer: An Exceptional Presentation of a Carcinomatous Mastitis
}

\author{
Saskia Venverloo ${ }^{a}$ Karl Wehbe $^{a}$ Eva Brabencova $^{b}$ Vivien Ceccato $^{a}$ \\ Judicael Hotton ${ }^{a}$ Clement Dabiri ${ }^{a}$ \\ aDepartment of Surgical Oncology, Institute Godinot, Reims, France; ${ }^{b}$ Department of \\ Pathology, Institute Godinot, Reims, France
}

Keywords
Advanced breast cancer · Carcinomatous mastitis - Ecchymotic purpura

\begin{abstract}
A 79-year-old woman was treated at our department for a neoplasm of the left breast. It was discovered following the spontaneous appearance of a localized ecchymotic lesion of the breast. The only clinical sign was this purpura, with no notion of trauma. The lesion was homogeneous, oval, and measuring $4 \times 5 \mathrm{~cm}$, and it was stable for 2 months. The patient had no history of thrombocytopenia and no known allergy. The physical examination was not very helpful and did not find any palpable breast lesion besides a 1-cm left axillary lymphadenopathy. Breast screening revealed a solid, poorly delineated structure of $11 \mathrm{~mm}$. Biopsies were taken and revealed a NOS grade II invasive carcinoma, triple negative, with a Ki-67 proliferation index of $15 \%$. The axillary cytology was positive. Faced with this atypical presentation, a skin biopsy was performed on the bruise in order to exclude skin invasion. Histology showed the presence of carcinomatous lymphatic dermal emboli which could correspond to a mammary origin as well as extravasation of blood in the dermis explaining this ecchymotic aspect. Bone scintigraphy found discrete uptake in the rib cage, spine, and pelvis, suspicious in this context, but a benign origin could not be ruled out. Neoadjuvant chemotherapy was initiated before mastectomy and left axillary node dissection. It was an atypical and rare clinical presentation of advanced breast cancer with no similar case found in the literature. Usually, the presence of emboli with carcinomatous cells obstructing the lymphatic vessels is related to inflammatory breast cancer or carcinomatous mastitis. The authors nevertheless point out that although the presence of tumor emboli in the dermal lymphatic vessels is favorable to the diagnosis, it is not required. In addition, dermal lymphatic invasion without a typical clin-
\end{abstract}


ical presentation is not sufficient to confirm the diagnosis of carcinomatous mastitis. An important diagnostic criterion is the rapidity of aggravation of the symptoms, which, even if it was not obvious in our case, was present with progressive evolution of the skin lesion over several weeks. The skin lesion of our patient could also make us suspect a primary breast angiosarcoma. This possibility was quickly eliminated in the absence of vascular tumor proliferation on the various biopsies. Any acute purpura is a serious disease to be diagnosed urgently, and a skin biopsy is indicated.

(C) 2021 The Author(s).

Published by S. Karger AG, Basel

\section{Introduction}

Breast cancer remains the most frequent and deadliest cancer for women in France and in most industrialized countries, despite significant progress in its early diagnosis and the biological characterization of the disease, as well as improvement of its management.

The prevalence of inflammatory breast cancer varies by country. In Europe, $6 \%$ of breast cancers are inflammatory cancers, also called carcinomatous mastitis. In North Africa, the prevalence varies between 5 and 10\%, while in the USA, it is around 2\% [1]. The diagnosis is primarily clinical, marked by the presence of thickening of the skin, nipple retraction, an orange peel appearance, and breast edema [2]. The diagnosis is clarified by breast imaging. It shows an aspect of circumferential or trabecular skin thickening, the presence of polymorphic microcalcification and an overall increase in breast density.

The diagnostic can be confirmed with the presence of emboli of carcinomatous cells obstructing the lymphatic channels found on a skin biopsy or even a lymphovascular dermal invasion [3]. It is essential to correctly stage these cancers before treatment, because this modifies the whole care process - in particular, surgical care.

\section{Case Report}

Ms. N. was a 79-year-old patient. Her main medical history included high blood pressure, cholelithiasis, and a lumpectomy of the left breast for benign adenosis. She was primiparous and menopausal from the age of 50 years and did not take any hormone replacement therapy. Her only family medical history was breast cancer in a sister.

This patient had consulted her general practitioner following the spontaneous appearance of a bruise on the left breast. A mammogram was then performed, which showed diffuse calcifications of the two breasts associated with a left breast cyst. Breast ultrasound confirmed the presence of the left cyst and showed a solid lesion of $11 \mathrm{~mm}$ in the left para-areolar region. The biopsy found a NOS grade II invasive carcinoma, triple negative, with a Ki-67 proliferation index of $15 \%$.

The patient was referred to our service for further care. She presented a purplish skin lesion in the inferior internal quadrant of the left breast. This lesion had appeared spontaneously with no notion of trauma or taking of any anticoagulant (Fig. 1). The patient's biological examination was normal and made it possible to eliminate idiopathic thrombocytopenic purpura and meningococcemia. In this context of an atypical presentation (ecchymotic purpura of unknown etiology), we performed a skin biopsy. The anatomopathological examination revealed the presence of triple-negative carcinomatous lymphatic emboli (Fig. 2, 3) with a Ki-67 proliferation index of 30\%, which may have corresponded to a breast origin, classifying the cancer as T4 according to the TNM classification.

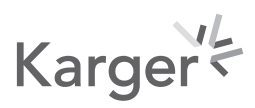


Fig. 1. Clinical aspect at the beginning of the initial assessment.

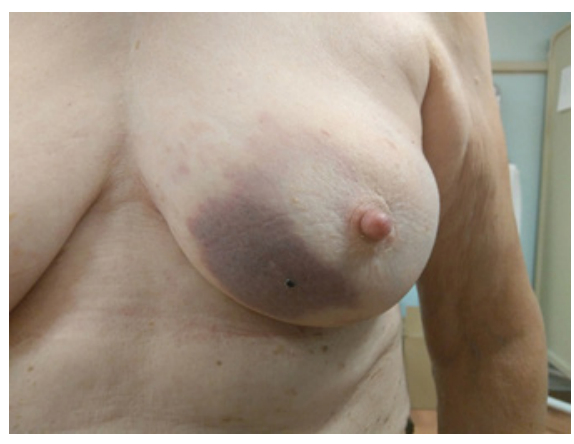

Fig. 2. Extravasation of blood in the dermis explaining the appearance of ecchymotic purpura.

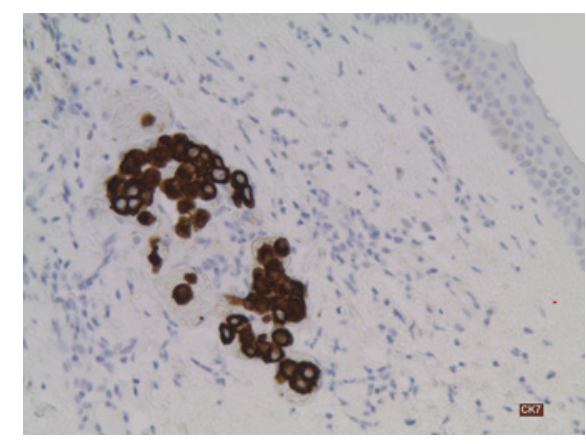

Fig. 3. Dermal lymphatic carcinomatous emboli.

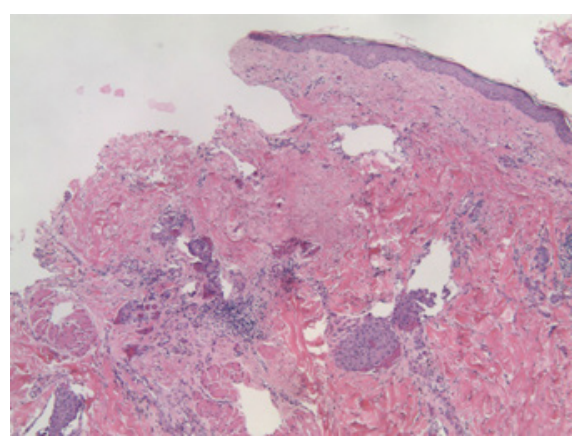

A second ultrasound was performed, showing an aspect of pseudocystic and partial tissue modification extending over $40 \mathrm{~mm}$. In addition, axillary lymphadenopathy was visualized, and cytology confirmed the malignancy.

A bone scan and whole-body CT scan were performed. They showed discrete hyperfixation foci in the ribs, spine, and pelvis, suspicious in this context, and also left axillary lymphadenopathy. After consultation with a dermatologist, we describe the lesion as follows: homogeneous purplish pseudo-ecchymotic infiltration of $65 \times 100 \mathrm{~mm}$ of the left breast located in the internal para-nipple area extending to the upper and lower levels bypassing the nipple. It was associated with a left lateral extension with brownish-to-orange appearance, which may correspond to an inflammatory cancer.

After discussion in a multidisciplinary meeting, the indication for neoadjuvant chemotherapy was retained. At that date, the ecchymotic lesion, initially located, now extended from the internal quadrants to the lower left quadrant. There was still a palpable lymphadenopathy in the left axillary region. The contralateral breast was free from any suspected clinical lesion.

Neoadjuvant chemotherapy was started with 4 cycles of doxorubicin and cyclophosphamide combined with primary prevention with Granocyte 34 every 3 weeks. Following 
Fig. 4. Clinical aspect after 4 cycles of doxorubicin and cyclophosphamide.
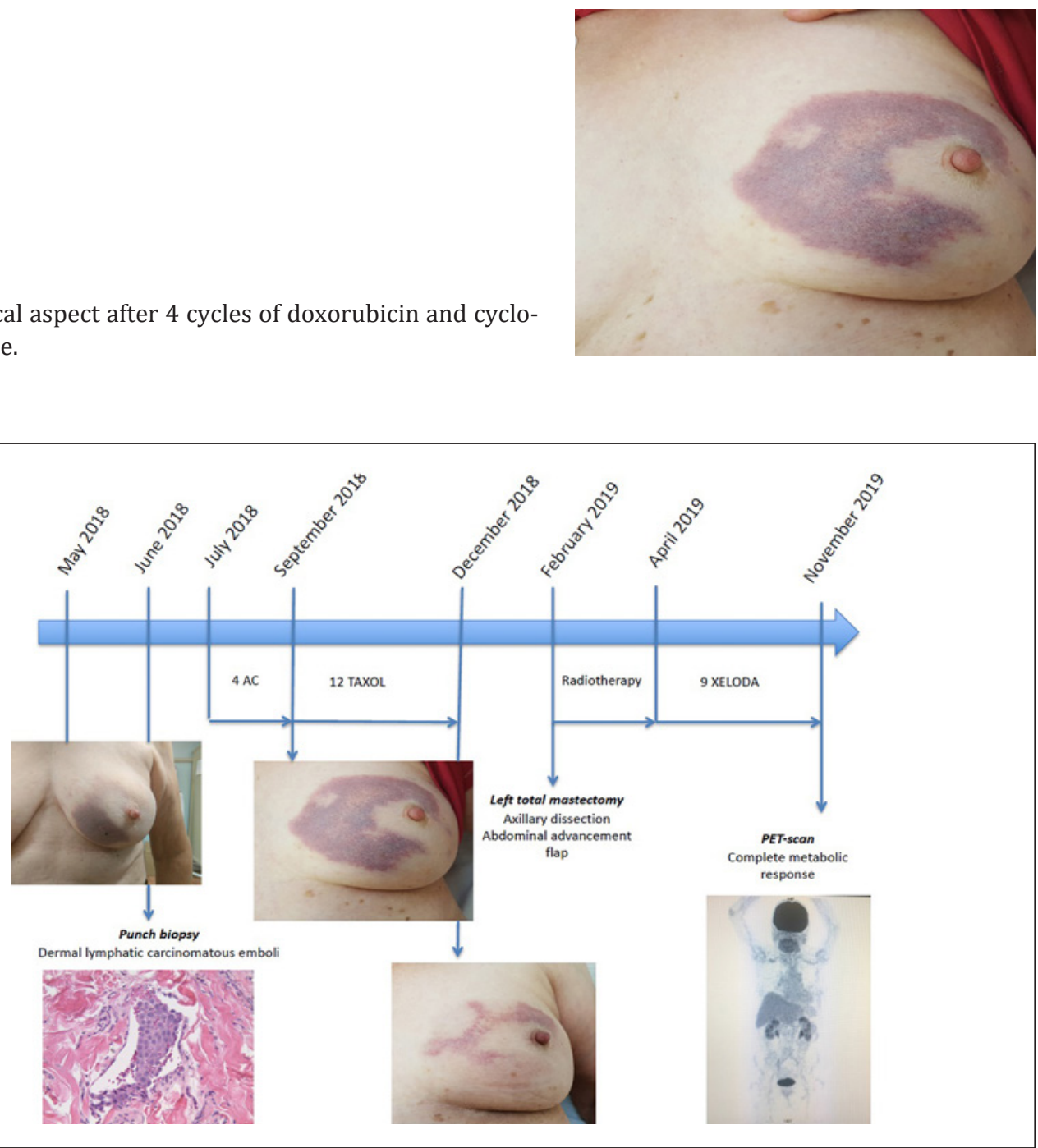

Fig. 5. Timeline of clinical and therapeutic evolution.

these well-tolerated treatments, we had observed a decrease in the purpuric lesion as well as a decrease in breast induration and a free axillary lymph node area (Fig. 4).

Thereafter, the patient received 12 Taxol injections weekly. At the end of the treatment, there was a disappearance of the cutaneous involvement with simple persistence of hyperpigmentation without palpable mass. The lymph node area remained free.

A re-evaluation after neoadjuvant chemotherapy was performed with an FDG-PET scan. Scintigraphy revealed discrete progression of the infracentimetric osteocondensations of the axial skeleton, without significant scintigraphic translation but remaining doubtful. The FDG-PET scan made it possible to confirm a complete metabolic response with absence of visualization of a suspected hypermetabolic focus over the entire explored volume of pelvicspinal osteocondensations of scar appearance.

Clinically, we observed the almost complete disappearance of the purpuric lesion after the various neoadjuvant chemotherapy treatments (Fig. 5). 
A total left mastectomy was then performed with a wide cutaneous excision associated with axillary dissection and an abdominal advancement flap, allowing a tension-free closure. The anatomopathological examination found a grade II plurifocal NOS invasive adenocarcinoma with high-grade ductal carcinoma in situ and abundant dermal lymphatic carcinomatous emboli.

In total, 3 tumors were found: in the upper internal quadrant $(9 \times 6 \mathrm{~mm})$, in the lower internal quadrant $(4 \mathrm{~mm})$, and at the union of the outer quadrants $(9 \times 8 \mathrm{~mm})$. Immunohistochemistry confirmed the triple-negative character with a Ki-67 proliferation index of 1\%. Twenty lymph nodes were assessed, of which 12 were invaded and 10 presented a capsular rupture classifying the neoplasia as ypT4ypN1.

The medical file was again discussed in the multidisciplinary meeting, offering adjunct locoregional radiotherapy, maintenance chemotherapy with capecitabine (Xeloda), and PET-FDG monitoring. A new evaluation by FDG-PET scan was performed after radiotherapy, and 6 treatments with capecitabine confirmed the maintenance of a complete metabolic response.

After the radiotherapy, there persisted a thoracic post-radiation hyperpigmentation and a grade 1 post-chemotherapy neuropathy. Two new treatments with Xeloda were performed, before the appearance of signs of poor tolerance required the treatment to be stopped. The last FDG-PET scan performed 17 months after the start of treatment confirmed the persistence of a complete metabolic response. We are continuing to monitor this patient by FDG-PET scan every 3 months.

\section{Discussion and Conclusion}

We reported an atypical and rare clinical presentation of breast cancer with no similar case found in the literature. Usually, the presence of emboli with carcinomatous cells obstructing the lymphatic vessels is related to inflammatory breast cancer (or carcinomatous mastitis) [4]. Some authors nevertheless point out that although the presence of tumor emboli in the dermal lymphatic vessels is favorable to the diagnosis, it is not required [3]. In addition, dermal lymphatic invasion without any typical clinical presentation is not sufficient to confirm the diagnosis of carcinomatous mastitis. Nevertheless, an important diagnostic criterion is the speed of aggravation of the symptomatology.

Here, the purpuric lesion was rapidly progressive (localized initially, and then extending almost to the entire breast a few weeks later). In our case, we could relate this clinical presentation to inflammatory breast cancer, aided by skin biopsy. The almost complete disappearance of the skin lesion after chemotherapy has confirmed our classification of this cancer as carcinomatous mastitis. In view of the severity of our case, which can be assessed using histological markers, we wonder if it would not seem wise to change the definition of inflammatory cancers so that this type of presentation is correctly diagnosed and managed. The skin lesion of our patient could also be suspicious for primary mammary angiosarcoma [5] or lymphangiosarcoma. Breast angiosarcomas are described by a soft, fast-growing breast tumor with opposite vascular signs, without satellite lymphadenopathy, and with a very important Doppler signal [6]. This possibility was quickly eliminated in the absence of vascular tumor proliferation on the different biopsies in our patient [7].

One of the elements which was not yet taken into account in our TNM classification, but which has already been described by the Gustave Roussy Institute, is the PEV (poussée évolutive) classification of active inflammation flares. They propose to classify inflammatory cancers as PEV1 with doubling of the tumor volume in 6 months, as PEV2 with inflammation of part of the breast, and as PEV3 with inflammation of the whole breast, also described as

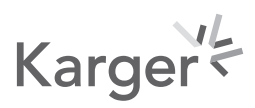




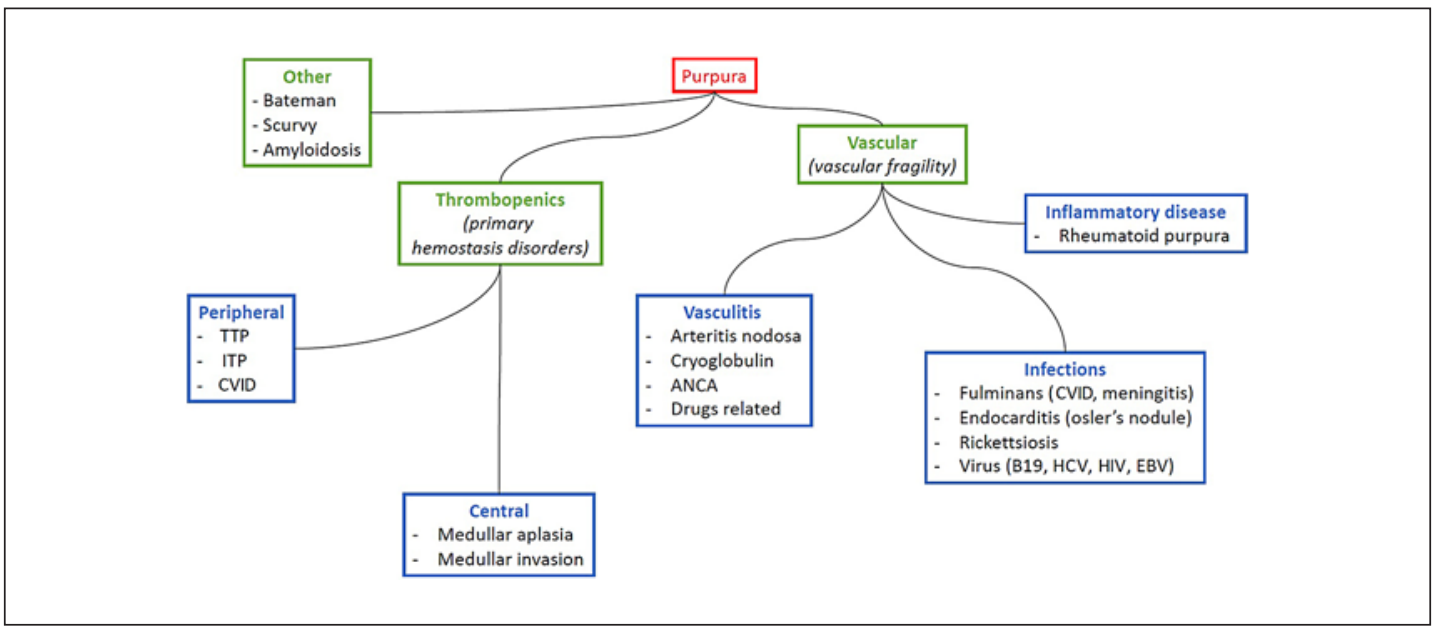

Fig. 6. Decision tree for purpura. TTP, thrombotic thrombocytopenic purpura; ITP, immune thrombocytopenic purpura; CVID, common variable immunodeficiency; ANCA, anti-neutrophil cytoplasmic autoantibody; B19, parvovirus B-19; HCV, hepatitis C virus; HIV, human immunodeficiency virus; EBV, Epstein-Barr virus.

carcinomatous mastitis. In our case, we could have classified our patient as PEV2, although, clinically, the breast was not strictly speaking inflamed but marked by a simple evolving purpura.

Any acute purpura is a port of call for a disease that can be serious, to be diagnosed as a matter of urgency so as not to overlook meningococcemia, severe thrombocytopenia, or infectious endocarditis. Skin biopsy is required when facing purpuric lesions of atypical presentation and in the absence of an obvious diagnosis [8] (Fig. 6).

The series by Fain et al. [9] described the diagnosis of solid tumors following the discovery of a purpuric lesion in the context of vasculitis. In their study, 60 cases of paraneoplastic vasculitis were described, 2 of which led to the diagnosis of breast cancer. This analysis is based on the importance of the search for neoplasia when facing a purpuric lesion; as in the present case, the appearance of atypical purpura must always lead to a search for cancer, most often hematological but in some cases of a solid origin. Our patient, who underwent a skin biopsy for a spontaneous breast ecchymotic lesion, was able to receive the adequate neoadjuvant treatment and the adapted surgical intervention.

The spontaneous appearance of purpura isolated from the breast is a clinical sign that should be suspicious for serious illness, especially if there is no prehistory of trauma or taking anticoagulants. It must raise suspicion for carcinomatous mastitis of atypical clinical presentation once other more frequent causes of purpura have been eliminated. In view of this clinical case, never described in the literature, we think that when facing an ecchymotic lesion of the breast, a complete senological assessment as well as skin biopsies in search of dermal carcinomatous emboli should be systematically carried out in order not to exclude any underlying and locally advanced breast neoplasia.

\section{Statement of Ethics}

Written informed consent was obtained from the patient for publication of this case report and any accompanying images. 


\section{Conflict of Interest Statement}

The authors have no conflicts of interest to declare.

\section{Funding Sources}

The authors have no specific grant from funding agencies in the public, commercial, or not-for-profit sectors.

\section{Author Contributions}

S.V. and C.D. collected data, designed the case report, and prepared and edited the manuscript. E.B. detailed the pathologic section and provided the histological illustrations. J.H. and K.W. assisted in editing the manuscript. All authors reviewed the manuscript.

\section{References}

1 Gunden JR, Bendahhou K, Benider A, Ibrahim Khalil A, Khalis M, Wilson ML, et al. Evaluating the incidence of inflammatory breast cancer using population- and hospital-based cancer registries in Casablanca, Morocco. Breast Dis. 2019;38(2):39-45.

2 Alunni JP. Imagerie du cancer du sein inflammatoire. J Radiol Diagn Intervent. 2012;93(2):103-11.

3 Mele M, Sørensen AS, Bruun J, Funder JA, Tramm T, Bodilsen A, et al. Inflammatory breast cancer: a review from our experience. Breast Dis. 2019;38(2):47-55.

4 van Uden DJ, van Laarhoven HW, Westenberg AH, de Wilt JH, Blanken-Peeters CF. Inflammatory breast cancer: an overview. Crit Rev Oncol Hematol. 2015;93(2):116-26.

5 Lyou Y, Barber E, Mehta R, Lee T, Goreal W, Parajuli R. Radiation-associated angiosarcoma of the breast: a case report and literature review. Case Rep Oncol. 2018;11(1):216-20.

6 Aljohani B, Al-Twajeri T, Alameer A, Alzaydi T, Alawwad S, Anwar I, et al. Clinicopathological features of breast angiosarcoma: a 16-years single-institution experience. Int J Surg Case Rep. 2017;37:211-5.

7 Nomoto Y, Kijima Y, Shinden Y, Hirata M, Eguchi Y, Yoshinaka H, et al. Two cases of radiation-associated angiosarcoma of the breast. Surg Case Rep. 2018;4(1):132.

8 Jung AJ, Schaeffer M, Mitcov M, Scrivener Y, Cribier B, Lipsker D. Étude anatomo-clinique du purpura: y a-t-il un intérêt à biopsier un purpura palpable?. Ann Dermatol Venereol. 2016;143(5):347-53.

9 Fain 0 , Hamidou M, Cacoub P, Godeau B, Wechsler B, Pariès J, et al. Vasculitides associated with malignancies: analysis of sixty patients. Arthritis Rheum. 2007;57(8):1473-80. 